\title{
The course and clinical correlates of panic disorder during the postpartum period: a naturalistic observational study
}

\author{
Semra Aydogan, ${ }^{1}$ Faruk Uguz, ${ }^{1}$ (iD Eda Yakut, ${ }^{2}$ Melike G. Bayman, ${ }^{2}$ Kazim Gezginc ${ }^{3}$ \\ ${ }^{1}$ Department of Psychiatry, University of Necmettin Erbakan, Meram Faculty of Medicine Konya, Konya, Turkey. ${ }^{2}$ Department of Psychiatry, \\ University of Health Sciences, Konya Training, Research Hospitals, Konya, Turkey. ${ }^{3}$ Department of Obstetrics and Gynecology, University of \\ Necmettin Erbakan, Meram Faculty of Medicine, Konya, Turkey.
}

\begin{abstract}
Objective: To investigate the course of panic disorder and its demographic and clinical correlates during the postpartum period.

Methods: Data were collected from 38 consecutive postpartum women diagnosed with panic disorder. Psychiatric assessments were carried out on the first day after delivery and at 6-8 weeks postpartum. During the first assessment, the Panic and Agoraphobia Scale (PAS), Hospital Anxiety and Depression Scale (HADS), Coping Orientation to Problems Experienced (COPE), Multidimensional Scale of Perceived Social Support (MSPSS), and Temperament Evaluation of Memphis, Pisa, Paris, and San Diego Autoquestionnaire (TEMPS-A) were administered to the participants. PAS was also administered at the second assessment.

Results: The mean PAS score reduced significantly from baseline to the second assessment. Logistic regression analysis indicated that a shorter duration of panic disorder independently predicted a $\geqslant 50$ point decrease in the severity of panic symptoms during the postpartum period.

Conclusion: These findings suggest that patients with a short duration of illness may experience significant alleviation in the severity of panic symptoms during the postpartum period.
\end{abstract}

Keywords: Pregnancy; panic disorder; postpartum period

\section{Introduction}

Pregnancy and the postpartum period are the most important reproductive events that can affect the physiological and mental status of women. Similar to other anxiety disorders, panic disorder is observed in women twice as frequently as in men. ${ }^{1-4}$ The assumption that reproductive life events may contribute to this higher prevalence in women via onset or recurrence of anxiety disorders has been supported by several studies. ${ }^{5-8}$

Panic disorder is an anxiety disorder that is frequently observed in the perinatal period. It has been reported that panic disorder during pregnancy is associated with lower birth weight and shorter gestational age.9,10 Moreover, a recent prospective-longitudinal study suggested that women with panic disorder during the peripartum or postpartum period reported a shorter duration of breastfeeding, impaired bonding, more loving/affection towards their infants, and more feeding problems in their infants. ${ }^{11}$ Therefore, when the potential negative effects of panic disorder on the fetus and children are considered, patients with this diagnosis should be closely monitored during the perinatal period. It is well known that the postpartum period may affect the course of psychiatric disorders. On the other

Correspondence: Faruk Uguz, Necmettin Erbakan Üniversitesi Meram Tıp Fakültesi, Psikiyatri Anabilim Dalı, Akyokuş, 42080, Konya, Turkey.

E-mail: farukuguz@gmail.com

Submitted Apr 19 2020, accepted Jul 18 2020, Epub Oct 232020. hand, detailed understanding of the natural course of psychiatric symptoms is an important factor in determining the treatment plan for patients during the postpartum period. Therefore, a highly pertinent question is how the postpartum period affects the severity of panic disorder in women. Several studies have already tried to address this question. Cohen et al. ${ }^{12}$ reported that 7 out of 10 patients experienced no meaningful change in clinical status between the third trimester of pregnancy and postpartum assessments; however, medication was increased in these patients after delivery. Earlier studies reported puerperal worsening of panic symptoms in $35-63 \%$ of patients. $^{13,14} \mathrm{~A}$ large-scale study by Bandelow et al. ${ }^{7}$ suggested that the proportion of women who experienced an increase in panic symptoms was $18 \%$. Contrary to the retrospective studies mentioned above, a prospective study with a small sample size showed that the severity of panic symptoms decreased considerably from pregnancy to 6 weeks postnatally. ${ }^{15}$ The authors proposed that the symptoms may have been ameliorated because of a decrease in social stress in most women; however, they also noted that stressful events and the level of social support were not examined. There are a limited number of prospective studies on this topic. Additionally, factors

How to cite this article: Aydogan S, Uguz F, Yakut E, Bayman MG, Gezginc K. The course and clinical correlates of panic disorder during the postpartum period: a naturalistic observational study. Braz J Psychiatry. 2021;43:6-11. http://dx.doi.org/10.1590/1516-44462020-1050 
related to exacerbation or alleviation of panic symptoms during the postpartum period have not been adequately examined in the literature. In this context, we aimed to investigate the following two questions: 1) what is the natural course of panic disorder during the postpartum period?; and 2) which sociodemographic and clinical factors are associated with significant alterations in symptom severity in women with panic disorder during the postpartum period?

\section{Methods}

\section{Sample}

The current study was conducted in women who delivered at the obstetric inpatient clinic of a university hospital. The sample was composed of 38 consecutive patients with panic disorder who met the inclusion and exclusion criteria. A diagnosis of panic disorder according to a structured psychiatric interview, age between 18 and 45 years, and voluntary participation in the study were the inclusion criteria. Patients with a diagnosis of bipolar disorder, schizophrenia and related psychotic disorders, mental retardation, multiple pregnancies, significant gestational complications (e.g., preeclampsia, gestational diabetes mellitus, and premature rupture of membranes), or severe medical problems, including endocrine, cardiovascular, pulmonary, metabolic, and neurological disorders, were excluded from the study. The study sample also did not include patients who used psychotropic medications, alcohol, or illicit drugs during pregnancy or the postpartum period. Neonatal medical conditions (e.g., neonatal septicemia, neonatal seizure, metabolic disturbances, and intracranial events) and congenital malformations in the infants were also considered exclusion criteria, as they can affect the course of maternal panic disorder by imposing additional stress and anxiety during the follow-up period.

\section{Assessments}

Panic disorder and other psychiatric disorders were diagnosed with the Structured Clinical Interview for the Diagnostic and Statistical Manual of Mental Disorders, Fourth Edition (SCID-I). ${ }^{16}$ Severity of panic symptoms and levels of anxiety and depressive symptoms were assessed with the Panic and Agoraphobia Scale $(\mathrm{PAS})^{17}$ and Hospital Anxiety and Depression Scale (HADS), ${ }^{18}$ respectively. To assess strategies for coping with psychosocial stresses and the level of social support of the participants, the Coping Orientation to Problems Experienced (COPE) ${ }^{19}$ and Multidimensional Scale of Perceived Social Support (MSPSS), ${ }^{20}$ respectively, were administered. In addition, the Temperament Evaluation of Memphis, Pisa, Paris, and San Diego Autoquestionnaire (TEMPS-A) ${ }^{21}$ was used to evaluate temperament characteristics.

\section{Procedures}

Sociodemographic characteristics and obstetric features were recorded at the obstetric inpatient clinic. The first psychiatric evaluation was performed within the first day following delivery. Structured psychiatric interviews were conducted by psychiatrists with at least 4 years of experience in psychiatric disorders and the use of diagnostic instruments. During the first interview, the patients were administered the SCID-I, PAS, HADS, COPE, MSPSS, and TEMPS-A. The second interview was completed at 6 to 8 weeks after delivery, when only the PAS was administered. The timing of the second interview was chosen because changes in the severity of psychiatric disorders are often observed during this early postpartum period. ${ }^{8,15}$ Both interviews were carried out by the same psychiatrist for each patient. The present study had a naturalistic observational design; therefore, there was no interference with the treatments being received by the participants of this study. Of note, based on the set exclusion criteria, only those women who did not receive any treatment during the study period were included.

\section{Statistical analyses}

The data were analyzed using SPSS for Windows version 16.0. Normally and non-normally distributed continuous variables were compared between the independent groups with the $t$-test and Mann-Whitney $U$ test, respectively. The PAS scores at the first and second evaluation were compared with the $t$-test for dependent groups. Categorical variables were compared among study groups using the chi-square $\left(\chi^{2}\right)$ test and, if necessary, Fisher's exact test. To determine independent factors related to a decrease of at least $50 \%$ in the PAS score, binary logistic regression analysis was performed. The significance level was set at 0.05 (all tests two-tailed).

\section{Ethics statement}

Initially, all participants were informed about the study and voluntary written informed consent was obtained. The ethics committee of the university hospital where the study was conducted approved the study procedures.

\section{Results}

The mean age of the sample was $30.47 \pm 6.07$ years. All participants were married and most were housewives $(n=37,97.4 \%)$, primary school $(n=30,78.9 \%)$ or secondary school $(n=6,15.8 \%)$ graduates, and multigravidae $(n=33,86.8 \%)$. Twelve $(31.6 \%)$ of the patients had a history of abortion. The average number of children was $2.45 \pm 0.83$. Twenty $(52.6 \%)$ of the infants born were female. Family history of panic disorder was positive in $13(34.2 \%)$ patients. Symptoms of panic disorder had existed before pregnancy in $24(63.2 \%)$ patients. Among the patients, $44.7 \%(n=17)$ had comorbid major depression or anxiety disorder during their pregnancy. Accordingly, the most common comorbid diagnoses were generalized anxiety disorder $(n=11,28.9 \%)$ and major depression ( $n=6,15.8 \%)$. During previous postpartum periods, a worsening of symptoms was reported by five $(38.5 \%)$ patients, a decrease was reported by five $(38.5 \%)$ patients, and no change was reported by 3 (23.1\%) patients. 
The second interview was carried out a mean of $6.78 \pm 0.81$ weeks postpartum. The PAS scores at the first and second interview were $26.31 \pm 8.33$ and 15.13 \pm 11.94 , respectively $(p=0.000)$. From baseline to 6 to 8 weeks postnatally, the PAS score decreased by at least $50 \%$ in $21(55.3 \%)$ patients. A decrease in scores of $\geqslant 25 \%$ but $<50 \%$ was found in $3(7.9 \%)$ patients. Symptom severity on the basis of PAS remained unchanged in the other patients $(n=14,36.8 \%)$.

For statistical comparison, patients who were classified into two groups based on the changes in their PAS scores: 1) patients who showed a decrease $\geqslant 50 \%$ in the PAS score at 6-8 weeks postnatally (decreased group); 2) patients who did not show the same level of decrease in the PAS score (non-decreased group). As shown in Table 1, differences between these groups for age, educational level, marital status, number of children, sex of the baby, gestational age, birth weight, and family history of panic disorder were not statistically significant. The proportion of patients with a history of abortion was significantly higher in the decreased group $(47.6 \%$ vs. $11.8 \%, p=0.034$ ) compared to the non-decreased group. Table 2 shows the symptom severity, disease duration, and comorbid diagnoses in the two groups. Statistical analyses indicated no significant differences between the groups for presence of comorbid depressive or anxiety disorders or PAS score at baseline. Patients in the non-decreased groups had a higher baseline score of HADS-Anxiety, HADS-Depression, and HADS-Total, and a longer duration of panic disorder. A total of 13 patients who had previously given birth experienced panic disorder before the delivery. The proportion of patients who reported a decrease in symptom severity following a previous childbirth in the decreased group and non-decreased group were $62.5 \%$ $(n=5)$ and $0 \%$, respectively; however, the difference was not statistically significant (Fisher's exact test, $p=0.075$ ). The MSPSS, COPE, and TEMPS-A scores of the two groups are presented in Table 3 . The levels of perceived social support were similar between the groups. The active coping subscale scores of COPE, but no other subscale scores, were significantly higher in the decreased group compared to the non-decreased group. In contrast, patients in the non-decreased group had higher TEMPS-A depressive and anxious subscale scores compared to the decreased group.

Variables such as history of abortion, duration of panic disorder, HADS-Total score, subscale scores of COPEActive coping, TEMPS-A-Depressive, and TEMPS-AAnxious were entered in a logistic regression analysis to determine the independent predictors of a decrease of at least $50 \%$ in PAS score from baseline to 6-8 weeks postnatally. Among these variables, the single independent predictor for at least $50 \%$ decrease in the severity of symptoms during the postpartum period was the duration of panic disorder (Wald $\chi^{2}=4.91$, degrees of freedom [df] $=1, \operatorname{Exp}(B)(95 \%$ confidence limits $[95 \% C l])=0.97$ [0.95-0.99], $p=0.027)$. The predictive values of history of abortion (Wald $\chi^{2}=3.13, \mathrm{df}=1, \operatorname{Exp}(\mathrm{B})[95 \% \mathrm{Cl}]=$ 0.07 [0.01-1.32], $\mathrm{p}=0.077$ ), HADS-Total score (Wald $\chi^{2}=0.53, \mathrm{df}=1, \operatorname{Exp}(\mathrm{B})[95 \% \mathrm{Cl}]=0.92[0.74-1.14]$, $\mathrm{p}=0.464$ ), subscale scores of COPE-Active coping (Wald $\chi^{2}=3.28, \mathrm{df}=1, \operatorname{Exp}(\mathrm{B})[95 \% \mathrm{Cl}]=1.44$ [0.97-2.15], $\mathrm{p}=0.070$ ), TEMPS-A-Depressive (Wald $\chi^{2}=0.41$, $\mathrm{df}=1$, $\operatorname{Exp}(\mathrm{B})[95 \% \mathrm{Cl}]=0.89[0.63-1.26], \mathrm{p}=0.520)$, and TEMPS-A-Anxious (Wald $\chi^{2}=0.14$, df $=1, \operatorname{Exp}(B)[95 \%$ $\mathrm{Cl}]=0.96[0.80-1.16], \mathrm{p}=0.713$ ) were not found to be statistically significant.

Table 1 Sociodemographic characteristics of patient groups with and without a decrease of at least $50 \%$ in severity of panic symptoms during the postpartum period

\begin{tabular}{|c|c|c|c|}
\hline & Decreased group $n=21$ & Non-decreased group $n=17$ & $p$-value \\
\hline Age (years) & $30.81 \pm 6.07$ & $30.06 \pm 6.24$ & $0.889 *$ \\
\hline $\begin{array}{l}\text { Education } \\
\text { Primary school } \\
\text { Secondary school } \\
\text { University }\end{array}$ & $\begin{array}{l}17(81) \\
3(4.3) \\
1(4.8)\end{array}$ & $\begin{array}{c}13(76.5) \\
3(17.6) \\
1(5.9)\end{array}$ & $0.945^{\dagger}$ \\
\hline Employment status (unemployed) & $20(95.2)$ & $17(100)$ & 1.000 \\
\hline Family history & $6(28.6)$ & 7 (41.2) & 0.502 \\
\hline Number of children & $2.57 \pm 0.74$ & $2.29 \pm 0.92$ & $0.367^{\S}$ \\
\hline Gestational age (weeks) & $37.29 \pm 2.33$ & $37.94 \pm 2.98$ & $0.452 *$ \\
\hline Birth weight $(\mathrm{g})$ & $2,957 \pm 765$ & $3,031 \pm 625$ & $0.150 *$ \\
\hline Gravidity (primigravid) & $1(4.8)$ & $4(23.5)$ & \\
\hline History of abortion & $10(47.6)$ & $2(11.8)$ & $0.034^{\ddagger}$ \\
\hline $\begin{array}{l}\text { Sex of baby } \\
\text { Male }\end{array}$ & $9(42.9)$ & $9(52.9)$ & $0.385^{+}$ \\
\hline
\end{tabular}

Data presented as mean \pm standard deviation or $\mathrm{n}(\%)$.

* $t$ test; ${ }^{\dagger} \chi^{2}$ test; ${ }^{\star}$ Fisher's exact test; ${ }^{\S}$ Mann-Whitney $U$ test. 
Table 2 Clinical characteristics of patient groups with and without a decrease of at least $50 \%$ in severity of panic symptoms during the postpartum period

\begin{tabular}{lccc}
\hline Variable & Decreased group $\mathrm{n}=21$ & Non-decreased group $\mathrm{n}=17$ & $\mathrm{p}$-value \\
\hline Comorbid diagnoses & $8(38.1)$ & $9(52.9)$ & 0.513 \\
Major depression & $4(19.1)$ & $1(1.8)$ & 0.672 \\
Dysthymic disorder & $0(0)$ & $0.11 .8)$ & 0.193 \\
Obsessive-compulsive disorder & $3(14.3)$ & $0(0)$ & 0.238 \\
Phobias & $0(0)$ & $7(41.2)$ & - \\
Posttraumatic stress disorder & $0(0)$ & $4(23.5)$ & 0.167 \\
Generalized anxiety disorder & $4(19.0)$ & $8(47.1)$ & 1.000 \\
Any depressive disorder & $4(19.0)$ & & 0.318 \\
Any anxiety disorder & $6(28.6)$ & $9.88 \pm 3.44$ \\
HADS scores & & $12.29 \pm 4.07$ \\
Depression & $6.90 \pm 3.98$ & $22.18 \pm 6.42$ \\
Anxiety & $9.38 \pm 3.70$ & $27.01 \pm 8.71$ \\
Total & $16.29 \pm 6.26$ & $62.47 \pm 54.77$ \\
PAS score & $25.71 \pm 8.18$ & 0.020 \\
Duration of panic disorder, months & $18.95 \pm 24.34$ & 0.007 \\
\hline
\end{tabular}

Data presented as $\mathrm{n}(\%)$ or mean \pm standard deviation.

HADS = Hospital Anxiety and Depression Scale.

Table 3 COPE, TEMPS-A, and MSPSS scores in patients with and without a decrease of at least $50 \%$ in severity of panic symptoms during the postpartum period

\begin{tabular}{|c|c|c|c|}
\hline Variable & Decreased group $n=21$ & Non-decreased group $n=17$ & $\mathrm{p}$-value \\
\hline \multicolumn{4}{|l|}{ COPE scores } \\
\hline Positive reinterpretation and growth & $13.05 \pm 2.13$ & $12.53 \pm 2.34$ & 0.481 \\
\hline Mental disengagement & $9.05 \pm 2.72$ & $10.35 \pm 2.29$ & 0.124 \\
\hline Focus on and venting of emotions & $12.33 \pm 2.49$ & $13.29 \pm 2.44$ & 0.242 \\
\hline Using instrumental social support & $11.86 \pm 3.02$ & $12.41 \pm 2.78$ & 0.564 \\
\hline Active coping & $13.10 \pm 1.86$ & $11.12 \pm 3.03$ & 0.019 \\
\hline Denial & $7.57 \pm 2.46$ & $8.82 \pm 2.81$ & 0.152 \\
\hline Religious coping & $14.38 \pm 2.78$ & $14.71 \pm 1.92$ & 0.686 \\
\hline Humor & $7.14 \pm 3.42$ & $6.82 \pm 2.55$ & 0.752 \\
\hline Behavioral disengagement & $7.71 \pm 2.49$ & $8.00 \pm 2.95$ & 0.748 \\
\hline Restraint & $9.86 \pm 2.85$ & $9.76 \pm 2.65$ & 0.919 \\
\hline Use of emotional social support & $12.29 \pm 2.93$ & $12.65 \pm 2.26$ & 0.672 \\
\hline Substance use & $4.33 \pm 0.85$ & $4.18 \pm 0.72$ & 0.552 \\
\hline Acceptance & $10.86 \pm 2.47$ & $11.53 \pm 2.80$ & 0.438 \\
\hline Suppression of competing activities & $10.67 \pm 1.56$ & $10.88 \pm 2.42$ & 0.742 \\
\hline Planning & $12.10 \pm 2.73$ & $11.71 \pm 3.15$ & 0.686 \\
\hline Total & $156.90 \pm 11.32$ & $158.35 \pm 19.68$ & 0.778 \\
\hline \multicolumn{4}{|l|}{ TEMPS-A scores } \\
\hline Depressive & $7.19 \pm 3.94$ & $10.88 \pm 3.53$ & 0.005 \\
\hline Cyclothymic & $7.76 \pm 5.19$ & $9.18 \pm 5.27$ & 0.412 \\
\hline Hyperthymic & $6.38 \pm 4.31$ & $5.00 \pm 3.58$ & 0.298 \\
\hline Irritable & $4.52 \pm 3.64$ & $5.47 \pm 3.95$ & 0.448 \\
\hline Anxious & $11.14 \pm 6.5$ & $15.94 \pm 5.39$ & 0.020 \\
\hline \multicolumn{4}{|l|}{ MSPSS scores } \\
\hline Family & $22.57 \pm 6.13$ & $21.71 \pm 6.04$ & 0.666 \\
\hline Friends & $16.86 \pm 7.59$ & $15.65 \pm 7.17$ & 0.620 \\
\hline Significant other & $23.05 \pm 5.85$ & $21.29 \pm 5.48$ & 0.394 \\
\hline Total & $62.48 \pm 15.55$ & $58.65 \pm 10.69$ & 0.412 \\
\hline
\end{tabular}

Data presented as mean \pm standard deviation.

COPE $=$ Coping Orientation to Problems Experienced; MSPSS = Multidimensional Scale of Perceived Social Support; TEMPS-A =

Temperament Evaluation of Memphis, Pisa, Paris, and San Diego Autoquestionnaire.

\section{Discussion}

To the best of our knowledge, this is the first prospective naturalistic study examining demographic and clinical factors related to the course of panic disorder during the postpartum period. Our findings suggest that the severity of panic symptoms may decrease during the first 6-8 weeks of the postpartum period. Moreover, in this study, $55.3 \%$ of participants showed a decrease of at least $50 \%$ in symptom severity, which meets the response criteria of 
studies investigating efficacy of pharmacological treatments in panic disorder. ${ }^{22,23}$ These results are inconsistent with previous retrospective studies that reported no change or worsening of symptoms during the postpartum period in approximately $90 \%$ of patients with panic disorder. ${ }^{7,12,13,14}$ However, the samples of these studies were composed of patients with panic disorder who were admitted to outpatient psychiatry clinics. Additionally, these studies were based on chart reviews or retrospective recall by the patients themselves, not on objective standardized instruments. In contrast, the current study administered objective standardized instruments to patients who were admitted to an inpatient obstetric clinic. A retrospective study with a large sample $(n=93)$ by Bandelow et al. ${ }^{7}$ reported that the postpartum period was associated with a substantial increase in panic symptoms compared to the pregnancy period. In addition, in a prospective study, Cohen et al. ${ }^{12}$ found no reduction in the severity of panic symptoms in 9 out of 10 patients, and increased level of antipanic treatment in 7 out of 10 patients. Nevertheless, neither of these two studies were carried out in a sample composed of patients who delivered their babies at an inpatient obstetric clinic. In addition, the exclusion criteria of these studies did not include comprehensive medical or obstetric factors that could influence the mental status of the women. These factors can explain the difference between the findings of the present study and those of these two. On the other hand, consistent with our results, a small prospective study with a similar sample profile and methods as our study suggested that the patients experienced a significant improvement in the severity of panic disorder at 6 weeks postpartum. ${ }^{15}$

In the current study, as in previous clinical samples, a reduction of at least $50 \%$ in severity of panic disorder from baseline to the second interview was defined as a significant alleviation in symptoms. ${ }^{22,23}$ The symptom severity decreased by at least $50 \%$ or remained unchanged in $92.1 \%$ of the patients; therefore, we analyzed the factors associated with a decrease in at least $50 \%$ (but no exacerbation) in the symptoms during the postpartum period. The proportion of patients in the decreased group who reported a reduction in symptoms after a previous delivery was markedly higher compared to the nondecreased group $(62.5 \%$ vs. $0 \%)$. This result did not reach statistical significance, which could be due to the small sample size. To the best of our knowledge, there are no studies in the published literature examining the effects of stress coping strategies, level of perceived social support, and personality characteristics in the course of panic disorder during the postpartum period. Guler et al. ${ }^{15}$ speculated that favorable sociocultural aspects, especially increased family support in the early weeks after delivery, might explain the reduction in severity of panic symptoms in the patient population in which the study was conducted. Nevertheless, the findings of the present study suggest that these factors do not significantly affect the course of the disorder. In our sample, the only independent factor that was associated with a significant alleviation in symptoms was a shorter duration of panic disorder. However, this may not be specific for the postpartum period. It has been shown that longer course as well as earlier onset of panic disorder are associated with nonresponse to treatment. ${ }^{24}$

The following limitations that may affect the study results should be considered during interpretation of the data. First, the relatively small sample size is a major limitation. However, our sample was still larger than those of available prospective studies ${ }^{13,15}$ and comparable to those of retrospective studies ${ }^{13,14}$ published in the literature. Second, the present study was carried out in a single university hospital; therefore, the sample may not be representative of all postpartum women. Similarly, the results may not be generalized to patients with panic disorder who were admitted to outpatient psychiatry clinics because the sample included patients who were admitted to an inpatient obstetric clinic. Third, the follow-up period included the early postpartum period; severity of panic symptoms may differ at the later stages of the postpartum period as well. Fourth, history of the course of panic symptoms after a previous childbirth was based on retrospective recall by the patients themselves. Fifth, we did not examine changes in diagnostic status and diagnostic severity across the two assessment points. On the other hand, the current study provides the most comprehensive information to date on the natural course and clinical correlates of symptom severity of panic disorder following childbirth.

In conclusion, despite the limitations mentioned above, the present study suggests that a significant decline in the severity of panic disorder symptoms may occur naturally in the postpartum period in about half of women. Moreover, if the duration of panic disorder is short, there is a greater likelihood of symptom relief. Therefore, these patients may require less frequent pharmacological treatment for panic disorder during the early postpartum period. If future prospective studies with larger sample sizes confirm these findings, this factor should be considered in planning the management of panic disorder after delivery. However, the findings of the present study do not show that this decrease in panic symptoms continues beyond 8 weeks postpartum. Therefore, close monitoring is warranted during later stages of the postpartum period, when symptoms may be elevated in some patients. Multicenter studies with larger samples may provide stronger data to support the observations of the current study. Further research should also examine the impact of other psychosocial factors (such as stressful life events), functional impairment, and detailed individual history of the disorder (e.g., treatment response and hospitalization) in the course of panic disorder during the postpartum period.

\section{Disclosure}

The authors report no conflicts of interest.

\section{References}

$1 \mathrm{Bijl} \mathrm{RV,} \mathrm{Ravelli} \mathrm{A,} \mathrm{van} \mathrm{Zessen} \mathrm{G.} \mathrm{Prevalence} \mathrm{of} \mathrm{psychiatric} \mathrm{disorder} \mathrm{in}$ the general population: results of the Netherlands Mental Health Survey and Incidence Study (NEMESIS). Soc Psychiatry Psychiatr Epidemiol. 1998;33:587-95.

2 Faravelli C, Abrardi L, Bartolozzi D, Cecchi C, Cosci F, D'Adamo D, et al. The Sesto Fiorentino Study: point and one-year prevalences of 
psychiatric disorders in an Italian community sample using clinical interviewers. Psychother Psychosom. 2004;73:226-34.

3 Vicente B, Kohn R, Rioseco P, Saldivia S, Levav I, Torres S. Lifetime and 12-month prevalence of DSM-III-R disorders in the Chile psychiatric prevalence study. Am J Psychiatry. 2006;163:1362-70.

4 Goodman JH, Chenausky KL, Freeman MP. Anxiety disorders during pregnancy: a systematic review. J Clin Psychiatry. 2014;75:e1153-84.

5 Vulink NC, Denys D, Bus L, Westenberg HG. Female hormones affect symptom severity in obsessive-compulsive disorder. Int Clin Psychopharmacol. 2006;21:171-5.

6 Guler O, Kaya V, Gezginc K, Kayhan F, Cicek E, Sonmez O, et al. Pegnancy-onset panic disorder: incidence, comorbidity and associated factors. Noro Psikiyatr Ars. 2015;52:216-20.

7 Bandelow B, Sojka F, Broocks A, Hajak G, Bleich S, Rüther E. Panic disorder during pregnancy and postpartum period. Eur Psychiatry. 2006;21:495-500.

8 Martini J, Petzoldt J, Einsle F, Beesdo-Baum K, Höfler M, Wittchen $\mathrm{HU}$. Risk factors and course patterns of anxiety and depressive disorders during pregnancy and after delivery: a prospective-longitudinal study. J Affect Disord. 2015;175:385-95.

9 Bánhidy F, Ács N, Puhó E, Czeizel AE. Association between maternal panic disorders and pregnancy complications and delivery outcomes. Eur J Obstet Gynecol Reprod Biol. 2006;124:47-52.

10 Uguz F, Sahingoz M, Sonmez EO, Karsidag C, Yuksel G, Annagur $\mathrm{BB}$, et al. The effects of maternal major depression, generalized anxiety disorder, and panic disorder on birth weight and gestational age: a comparative study. J Psychosom Res. 2013;75:87-9.

11 Martini J, Boesdo-Baum K, Garthus-Niegel S, Wittchen HU. The course of panic disorder during the peripartum period and the risk for adverse child development: a prospective-longitidunal study. J Affect Disord. 2020;266:722-30.

12 Cohen LS, Sichel DA, Faraone SV, Robertson LM, Dimmock JA, Rosenbaum JF. Course of panic disorder during pregnancy and the puerperium: a preliminary study. Biol Psychiatry. 1996;39:950-4.
13 Cohen LS, Sichel DA, Dimmock JA, Rosenbaum JF. Postpartum course in women with preexisting panic disorder. J Clin Psychiatry. 1994;55:289-92.

14 Northcott CJ, Stein MB. Panic disorder in pregnancy. J Clin Psychiatry. 1994;55:539-42.

15 Guler O, Koken GN, Emul M, Ozbulut O, Geçici, Uguz F, et al. Course of panic disorder during the early postpartum period: a prospective analysis. Compr Psychiatry. 2008;49:30-4.

16 First MB, Spitzer RL, Gibbon M, Williams JBW. Structured clinical interview for DSM-IV clinical version (SCID-I/CV). Washington: American Psychiatric Press; 1997.

17 Bandelow B. Assesing the efficacy of treatments for panic disorder and agoraphobia. II. The panic and agoraphobia scale. Int Clin Psychopharmacol. 1995;10:73-81.

18 Zigmund AS, Snaith RP. The hospital anxiety and depression scale. Acta Psychiatr Scand. 1983;67:361-70.

19 Carver CS, Scheier MF, Weintraub JK. Assessing coping strategies: a theoretically based approach. J Pers Soc Psychol. 1989;56:267-83.

20 Zimet GD, Dahlem NW, Zimet SG, Farley GK. The Multidimensional scale of perceived social support. J Pers Assess. 1988;52:30-41.

21 Akiskal HS, Akiskal KK, Haykal RF, Manning JS, Connor PD. TEMPS-A: progress towards validation of a self-rated clinical version of the temperament evaluation of the Memphis, Pisa, Paris, and San Diego autoquestionnaire. J Affect Disord. 2005;85:3-16.

22 Choi KW, Woo JM, Kim YR, Lee SH, Lee SY, Kim EJ, et al. Longterm escitalopram treatment in Korean patients with panic disorder: a prospective, naturalistic, open-label, multicenter trial. Clin Psychopharmacol Neurosci. 2012;10:44-8.

23 Goddard AW, Mahmud W, Medlock C, Shin YW, Shekhar A. A controlled trial of quetiapine XR coadministration treatment of SSRIresistant panic disorder. Ann Gen Psychiatry. 2015;14:26.

24 Chen $\mathrm{MH}$, Tsai SJ. Treatment-resistant panic disorder: clinical significance, concept and management. Prog Neuropsychopharmacol Biol Psychiatry. 2016;70:219-26. 